\title{
Two stage management of long bone infection by using an antibiotic cement-coated locking plate: an outcome of 183 cases
}

\section{Chao Jia}

Southwest Hospital, Third Military Medical University(Army Medical University)

\section{Xiaohua Wang}

Southwest Hospital, Third Military University(Army Medical University)

\section{Shengpeng $\mathrm{Yu}$}

Southwest Hospital, Third Military Medical University(Army Medical University)

\section{Hongri Wu}

Southwest Hospital, Third Military Medical University (Army Medical University)

Jie Shen

Southwest Hospital, Third Military Medical University(Army Medical University)

zhao xie ( $\square$ xiezhao54981@163.com)

Third Military Medical University Southwest Hospital

\section{Research article}

Keywords: Antibiotic cement coated locking plate, Bone infection, Internal fixation

Posted Date: September 18th, 2019

DOI: https://doi.org/10.21203/rs.2.14572/v1

License: (1) This work is licensed under a Creative Commons Attribution 4.0 International License. Read Full License 


\section{Abstract}

Background The purpose of this study was to evaluate the clinical effect of antibiotic cement-coated locking plate as temporary internal fixation for the treatment of long bone infection in lower extremities .

Methods Retrospective analysis the clinical data of 183 patients with lower extremities long bone infection admitted to our hospital from January 2013 to December 2017. All patients were treated with locking plate internal fixation after primary debridement. Antibiotic cement was used to fill the bone defect and wrap the plate. After infection control, bone grafting to reconstruct the bone defect.

Results There were 81 cases of femur, 100 cases of tibia and 2 cases of fibula, after the first stage, $16(8.7 \%)$ patients had recurrence of infection after the first stage which have to debridement again. 4 tibial infecton patients had poor wound healing, 2 femoral patients had fixation failure, and 1 patient had femoral fracture due to fall down. After a mean time of 32.0 months (range 12 to 66) follow up, the recurrence rate of infection was $14.2 \%(26 / 183)$. Among them, eighteen patients underwent bone grafting after re-debridement, 6 patients received permanent placement of antibiotic bone cement after debridement, and 2 patients refused further treatment and chose amputation. Bone healing was achieved in $175(95.9 \%$ ) patients in the last follow-up, and the average time of bone union was 5.4 months (range 4 to 12 months).

Conclusions Temporary internal fixation with antibiotic cement coated locking plate has good clinical effect in the treatment of lower extremities long bone infection. It does not increase the difficulty of bone infection control. Pay attention to possible difficulty of skin coverage when applied in calves. Key words: Antibiotic cement coated locking plate, Bone infection, Internal fixation

\section{Background}

Bone infection is a major problem in the field of orthopedics. Sufficient stabilization of bone defects after thorough debridement is conducive to infection control. External fixation was considered as the gold standard for the treatment of bone infections[1], which can not only provide stability, but also avoid the presence of foreign bodies in the infected wound and prevent the formation of biofilms which leading to the recurrence of infection[2]. However, external fixation have inherent limitations and many complications, such as pin track infection[3], poor stability, joint stiffness, and some patients can not tolerate the long-term impact of wearing external fixation. In order to make up for the above deficiencies and shortcomings, some scholars have explored the conditions of using internal fixation in bone infection sites. Liporace FA[4] and Conway JD[5] attempted to treat infected bone fractures with antibioticimpregnated bone cement-coated locking plates, which was preliminarily reported, with small cases and short follow-up time. We report of long bone infection in lower extremities treated with locking plate internal fixation which coated with antibiotic cement, acquired good clinical efficacy for at least one years of follow up, so as to provide a reference for clinicians to apply this method. 


\section{Materials And Methods}

The study was approved by the Ethics Committee of our hospital, and informed consent was obtained from the patients for the publication of individual clinical details and accompanying images. Our inclusion criteria were the following: (1) patients with lower extremities long bone infection admitted to our hospital; (2) treated with induced membrane two-stage technique and fixed with antibiotic cementcoated locking plate as internal fixation after debridement; (3) reconstruction of bone defect with the induced membrane technique. Exclusion criteria were following: (1) Malignant tumors; (2) patients with vascular and nerve injuries in affected limbs; (3) follow-up time were less than 1 year; (4) with incomplete follow-up data.

From January 2013 to December 2017, 183 patients were enrolled, 154 of the patients were male and 29 were female. The average age was 42.8 years (range, $10-68$ years). The average follow-up duration was 32.0 months (range 12 to 66). Among them, there were 81 cases of femur, 100 cases of tibia and 2 cases of fibula. There were 35 cases of hematogenous osteomyelitis and 148 cases of post-traumatic osteomyelitis, 94 cases were open fracture and 54 cases were closed fracture. According to Cierny-Mader classification, there were 48 cases of type III and 135 cases of type VI. According to Cierny-Mader host classification, there were 109 patients were defined as type $A$ and 85 as type B. Bacteria were isolated in 113 patients and 69 with negative bacteria cultures, among the 113 patients, 92 cases were single bacterial infection and 21 cases were mixed bacterial infection. (data show in Table.1区

In the first stage, thoroughly debridement was performed, then internal fixation with locking plate and coated with antibiotic cement to stabilize the bone defect, filling the bone defect with antibiotic bone cement for the formation of induced membrane. In the second stage, bone graft to reconstruct the bone defect.

Determined the infection area according to X-rays, MRI and radionuclide examination. The sinus, sequestrum, inflammatory granulation tissue, ischemic sclerosis bone was thoroughly cleared. The medullary cavity was cleared by reaming drill until punctate hemorrhage appeared. The deep tissues were sampled at multiple sites for pathological examination and microbiological examination. A large amount of iodophor, hydrogen peroxide and saline were used to wash the operation area repeatedly during the operation. Bone tissue and soft tissue were continuously removed for $5 \mathrm{~mm}$ and $2 \mathrm{~mm}$ respectively. Locking compression plate was used to fix the femur and reconstruction locking plate was used to fix the tibia and fibula. The antibiotic cement $(0.5 \mathrm{~g}$ gentamicin per $40 \mathrm{~g}$ of PMMA mixed with $5 \mathrm{~g}$ vancomycin) was used to fill the defect area, wrap the plate and screw at the same time, and extend 1-2 cm to the proximal and distal ends to expand the volume of the induced membrane. Free flaps or local transfer flaps were used when it's hard to suture the section directly. Sensitive antibiotics were used after operation according to bacteria isolated, and third-generation cephalosporins were used in culturenegative patients. If the infection recurs, the first segment operation is done repeatly. Patients are encouraged to take the initiative to conduct joint exercise, full weight bearing of the affected limb is forbidden. 
At least 6 weeks after primary debridement, the soft tissue condition was good (no swelling, fever, pain and sinus tract), Leukocyte count, erythrocyte sedimentation rate (ESR) and C-reactive protein (CRP) returned to normal, and the imaging examination did not indicate infection, so the second stage operation was feasible. During the second stage surgery, the incision of the former was chosen and the induced membrane was cut longitudinally to protect the integrity of the induced membrane carefully. The bone cement and internal fixation were removed thoroughly, and the bone ends were decorticated. Autogenous cancellous bone was taken from iliac bone and allogenic bone was mixed for patients with large bone defect. Intramedullary nail, locking plate or intramedullary nail-assisted locking plate was selected for final fixation. The induced membrane was sutured carefully, and then the incision was sutured completely.

Follow-up were made every month after the first stage, and every three to six months after the second stage. The review included clinical symptoms and signs of affected limbs (such as swelling, fever, pain and sinus formation), laboratory examinations (Leukocyte count, ESR, CRP), and anteroposterior and lateral X-rays. The recurrence of infection, bone healing and complications were observed and recorded. When the Leukocyte count, ESR and CRP were normal and there was no clinical manifestation of local infection (such as swelling, fever, pain and sinus formation), no recurrence of infection was considered. The bone union time was obtained by X-ray, radiological union was defined as three-sided cortical bridging on two perpendicular X-rays of the defect zone[6].

\section{Statistics}

Data analysis was performed using SPSS 23.0 statistical software (SPSS Inc, USA). The measurement data is represented with ranges and the enumeration data is expressed by rate. Enumeration data were compared using Pearson or Continuity Correction chi-square test. and the test level a value is 0.05 on both sides.

\section{Results}

After the first stage, 16 (8.7\%) cases had recurrence of infection, among them, 12 cases underwent two times debridement, 4 cases underwent three times debridement, and infection was controlled finally. Complications occurred in 7(3.8\%) cases: delayed union of tibial incision in 4 cases; complications related to internal fixation in 3 cases: non-infectious femoral fracture in 1 case due to falls; and fixation failure in 2 cases of long segmental bone defect in the middle femur. There was significant difference in the recurrence rate between type $A(4 / 103)$ and type $B(12 / 68)$ patients after primary surgery $(P<0.05)$. There was no significant difference in recurrence rate among different sites of bone infection $(P>0.05)$. (data show in table.2)

After a mean follow-up period of 32.0 months (range 12 to 66 months), 26 (14.2\%) cases recurred of infection, after debridement, permanent antibiotic cement was placed without bone reconstruction in 6 
patients. Two patients refused further debridement and opted for amputation. 175 patients (95.6\%) obtained radiographic bone union, in average 5.4 months (range 4 to 12 months).

\section{Discussion}

Bone infection is a major problem in the field of orthopedics. Its essence is bacterial biofilms[7], which can resist host immunity and antibiotics $[8,9]$. Bacteria could easily adhere to the surface of metal surface and formation of biofilm which leads to infection recurrence[10, 11]. Therefore, internal fixation is not recommended for bone infections. As widely accepted instrument, external fixator can span the infected area, avoid the exposure of metal foreign bodies, and prevent the formation of biofilms[12]. However, the high incidence of complications such as pin track infection limited its advantage[3]. When the infected site is close to the joint, trans-joint fixation leads to joint stiffness[13], which is not conducive to limb function recovery. The external fixator is bulky and difficult to wear, which affects patients' ordinary life[14]. Long-term wearing of the external fixator also has a negative impact on patients' mental health [15]. Mark[16] reported 11 cases of femur infection treated with plate internal fixation after complete debridement. Alemdar[17] reported that 15 cases of deep infection after lower limb fracture were treated with "non-contact" plate internal fixation on the basis of complete debridement, but no matter how thorough debridement, bacteria still existed[18], residual bacteria formed biofilm on the surface of the internal fixator, resulting in recurrence of infection.

Wrapping with antibiotic bone cement can prevent bacteria from adhering to the surface of the internal fixation, and the antibiotics released from antibiotic bone cement can kill the residual bacteria after debridement, preventing the internal fixation from becoming a new source of infection[19]. Our research applied internal fixations with locking plate which coated with antibiotic bone cement for the treatment of long bone infection. The infection control rate after the first debridement was $91.3 \%$, breaking through the prohibition of internal fixation in the case of infection. The premise is complete debridement of the bone and soft tissue, followed by good soft tissue coverage, therefore, thorough debridement is the core for bone infection treatment[20,21]. The ideal state of bone and soft tissue can be achieved by thorough debridement, so that the blood supply of tissue provide normal physiological function. The formation of biofilm is prevented by systemic antibiotics and local antibiotics[22-25]. At the same time, rigid stability is also an important factor for bone infection control[26, 27]. Animal experiments confirmed that the infection rate in the unstable group was more than twice as high as that in the stable group[28]. The locking plate provides angular stability and axial stability[29], which provides excellent anchoring in osteoporotic bone, it can also help protect joint function by avoiding trans-articular fixation when bone infection occurs in the metaphysis. In addition, locking plate does not contact with bone cortex and helps to protect periosteal blood supply.

In this study, a total of 183 patients were treated with antibiotic cement coated locking plate. After the first debridement, 16 (8.7\%) patients had recurrence of infection, which was manifested as wound nonunion, sinus, and local limb redness, swelling and pain. After postoperative review, the main cause of recurrence was incomplete debridement. After repeated debridement, the infection was controlled and 
bone reconstruction was carried out smoothly. This study found that the recurrence rate of type $B(17.6 \%)$ after debridement was significantly higher than that of type A patients $(3.9 \%)(P<0.05)$. This finding is consistent with Simpson's prospective study. Simpson[30] found that patients defined as host $B$ had a higher recurrence rate than patients defined as host $A$, and that host $B$ patients were recommended for more extensive debridement. All 4 cases of local skin delayed union occurred in the lower leg, the condition of local soft tissue is bad due to infection and repeated surgery, resulted in greater tension of incision suture. Therefore, skin flaps are recommended to ensure tension-free suture of incision. Limb elevation and anti-swelling drugs were used after operation. Fracture or loosening of internal fixation is mainly related to weight bearing and accidental falls. Therefore, patients are recommended to walk with crutches without weight bearing or partial weight bearing after the first stage.

The induced membrane technique[31] proposed by Masquelet in 2000 is a new method for the treatment of bone defects, the membrane prevents the absorption of the graft bone and produces factors that promote osteogenesis[32], and the bone union rate is $80 \% \sim 100 \%[33]$. In this study, 175(95.6\%) patients obtained radiological bone union, and the union time was 5.4 months (range 4 to 12 months). The plate was completely wrapped in the bone cement ensured the integrity of the induced membrane. Furthermore, the coverage is extended to the bone tip to increase the volume of the induced membrane. Bone cement releases high concentration of antibiotics, it can induce a highly vascularized membrane rich in various bone factors[34], which provides a good environment for bone reconstruction. In the second stage, the integrity of the induced membrane should be protected, which is very important for bone healing.

Although this study has a good success rate and breaks through many traditional theoretical and technical taboos, there are still some deficiencies. Firstly, this study is a retrospective study, it can only be summarized from clinical experience for some postoperative complications, but not confirmed from the experiment. Secondly, there is no control group, and no comparison with other fixed methods.

\section{Conclusions}

Temporary internal fixation with antibiotic cement coated locking plate has good clinical effect in the control of lower extremities long bone infection, and is practical in all parts of the lower extremities, but the application premise must be thoroughly debridement, at the same time, attention should be paid to the skin coverage of the lower leg.

\section{Declarations}

\section{Abbreviations}

MRSA: Methicillin-resistant Staphylococcus aureus; ESR: erythrocyte sedimentation rate; CRP:C-reactive protein; MRI: Magnetic resonance imaging; PMMA: Polymethyl methacrylate. 


\section{Ethics approval and consent to participate}

The study was authorized by the Medical Ethics Committee of Southwest hospital (Ref. No. KY201878). All patients or patient's legal guardian gave written informed consent for inclusion in the study.

\section{Consent for publication}

Consent to publish was obtained for the patients or patient's legal guardian whose information appears in this publication.

\section{Availability of data and materials}

The datasets generated and/or analyzed during the current study are not publicly available due protection patients' information but are available from the corresponding author on reasonable request.

\section{Competing interests}

The authors declare that they have no competing interests.

\section{Funding}

This work was supported by The National Natural Science Foundation of China (81672160); The National Key Research and Development Program of China (2016YFC1102005).

\section{Authors' contributions}

CJ and XHW wrote the manuscript and performed the data collection. SPY and HRW performed data collection, made figures. JS performed the data interpretation, and helped to write the manuscript. All authors read and approved the final manuscript. CJ and XHW contributed equally to this work. ZX designed and performed the study.

\section{Acknowledgements}

Not applicable. 


\section{References}

1.Cierny G, 3rd, Mader JT, Penninck JJ: A clinical staging system for adult osteomyelitis. Clin Orthop Relat Res 2003(414):7-24.

2.Giannoudis PV: Treatment of bone defects: Bone transport or the induced membrane technique? Injury 2016, 47(2):291-292.

3.Parameswaran AD, Roberts CS, Seligson D, Voor M: Pin tract infection with contemporary external fixation: how much of a problem? J Orthop Trauma 2003, 17(7):503-507.

4.FA囚 L, Yoon RS, Frank MA: Use of an "antibiotic plate" for infected periprosthetic fracture in total hip arthroplasty. J Orthop Trauma 2012, 26(3):18.

5.Conway JD, Hlad LM, Bark SE: Antibiotic cement-coated plates for management of infected fractures. Am J Orthop (Belle Mead NJ) 2015, 44(2):E49-53.

6.Blum AL, BongioVanni JC, Morgan SJ, Flierl MA, dos Reis FB: Complications associated with distraction osteogenesis for infected nonunion of the femoral shaft in the presence of a bone defect: a retrospective series. The Journal of bone and joint surgery British volume 2010, 92(4):565-570.

7.Zimmerli W, Sendi P: Orthopaedic biofilm infections. APMIS: acta pathologica, microbiologica, et immunologica Scandinavica 2017, 125(4):353-364.

8.Trampuz A, Zimmerli W: Diagnosis and treatment of infections associated with fracture-fixation devices. Injury 2006, 37 Suppl 2:S59-66.

9.Costerton JW: Bacterial Biofilms: A Common Cause of Persistent Infections. Science 1999, 284(5418):1318-1322.

10.Parsons B, Strauss E: Surgical management of chronic osteomyelitis. The American Journal of Surgery 2004, 188(1):57-66.

11.Evans RP, Nelson CL, Harrison BH: The effect of wound environment on the incidence of acute osteomyelitis. Clin Orthop Relat Res 1993(286):289-297.

12.Mauffrey C, Hake ME, Chadayammuri V, Masquelet AC: Reconstruction of Long Bone Infections Using the Induced Membrane Technique: Tips and Tricks. J Orthop Trauma 2016, 30(6):e188-193.

13.Masquelet AC, Kishi T, Benko PE: Very long-term results of post-traumatic bone defect reconstruction by the induced membrane technique. Orthop Traumatol Surg Res 2019, 105(1):159-166.

14.Wang Y, Jiang H, Deng Z, Jin J, Meng J, Wang J, Zhao J, Sun G, Qian H: Comparison of Monolateral External Fixation and Internal Fixation for Skeletal Stabilisation in the Management of Small Tibial Bone 
Defects following Successful Treatment of Chronic Osteomyelitis. BioMed research international 2017, 2017:6250635.

15.Abulaiti A, Yilihamu Y, Yasheng T, Alike Y, Yusufu A: The psychological impact of external fixation using the llizarov or Orthofix LRS method to treat tibial osteomyelitis with a bone defect. Injury 2017, 48(12):2842-2846.

16.Prasarn ML, Ahn J, Achor T, Matuszewski P, Lorich DG, Helfet DL: Management of infected femoral nonunions with a single-staged protocol utilizing internal fixation. Injury 2009, 40(11):1220-1225.

17.Alemdar C, Azboy I, Atic R, Ozkul E, Gem M, Kapukaya A: Management of infectious fractures with "Non-Contact Plate" (NCP) method. Acta Orthop Belg 2015, 81(3):523-529.

18.Kinik H, Karaduman M: Cierny-Mader Type III chronic osteomyelitis: the results of patients treated with debridement, irrigation, vancomycin beads and systemic antibiotics. International orthopaedics 2008, 32(4):551-558.

19.Campoccia D, Montanaro L, Arciola CR: The significance of infection related to orthopedic devices and issues of antibiotic resistance. Biomaterials 2006, 27(11):2331-2339.

20.Hogan A, Heppert VG, Suda AJ: Osteomyelitis. Archives of orthopaedic and trauma surgery 2013, 133(9):1183-1196.

21.Waldvogel FA, Papageorgiou PS: Osteomyelitis: the past decade. N Engl J Med 1980, 303(7):360-370.

22.Pan C, Zhou Z, Yu X: Coatings as the useful drug delivery system for the prevention of implant-related infections. J Orthop Surg Res 2018, 13(1):220.

23. Hake ME, Young H, Hak DJ, Stahel PF, Hammerberg EM, Mauffrey C: Local antibiotic therapy strategies in orthopaedic trauma: Practical tips and tricks and review of the literature. Injury 2015, 46(8):1447-1456.

24.Zalavras CG, Patzakis MJ, Holtom P: Local antibiotic therapy in the treatment of open fractures and osteomyelitis. Clin Orthop Relat Res 2004(427):86-93.

25.Thonse R, Conway J: Antibiotic cement-coated interlocking nail for the treatment of infected nonunions and segmental bone defects. Journal of Orthopaedic Trauma 2007, 21(4):258-268.

26.Patzakis MJ, Wilkins J, Wiss DA: Infection following intramedullary nailing of long bones. Diagnosis and management. Clin Orthop Relat Res 1986(212):182-191.

27.Chen CE, Ko JY, Wang JW, Wang CJ: Infection after intramedullary nailing of the femur. Journal of Trauma-Injury Infection and Critical Care 2003, 55(2):338-344. 
28. Worlock P, Slack R, Harvey L, Mawhinney R: The prevention of infection in open fractures: an experimental study of the effect of fracture stability. Injury 1994, 25(1):31-38.

29.Wagner M: General principles for the clinical use of the LCP. Injury 2003, 34:31-42.

30.Simpson AH, Deakin M, Latham JM: Chronic osteomyelitis. The effect of the extent of surgical resection on infection-free survival. The Journal of bone and joint surgery British volume 2001, 83(3):403-407.

31.Masquelet AC, Fitoussi F, Begue T, Muller GP: Reconstruction of the long bones by the induced membrane and spongy autograft. Ann Chir Plast Esthet 2000, 45(3):346-353.

32.Chotel F, Nguiabanda L, Braillon P, Kohler R, Berard J, Abelin-Genevois K: Induced membrane technique for reconstruction after bone tumor resection in children: a preliminary study. Orthop Traumatol Surg Res 2012, 98(3):301-308.

33.Morris R, Hossain M, Evans A, Pallister I: Induced membrane technique for treating tibial defects gives mixed results. The bone \& joint journal 2017, 99-b(5):680-685.

34.Aho OM, Lehenkari P, Ristiniemi J, Lehtonen S, Risteli J, Leskela HV: The mechanism of action of induced membranes in bone repair. J Bone Joint Surg Am 2013, 95(7):597-604.

\section{Tables}

Due to technical limitations, tables are only available as a download in the supplemental files section

\section{Figures}
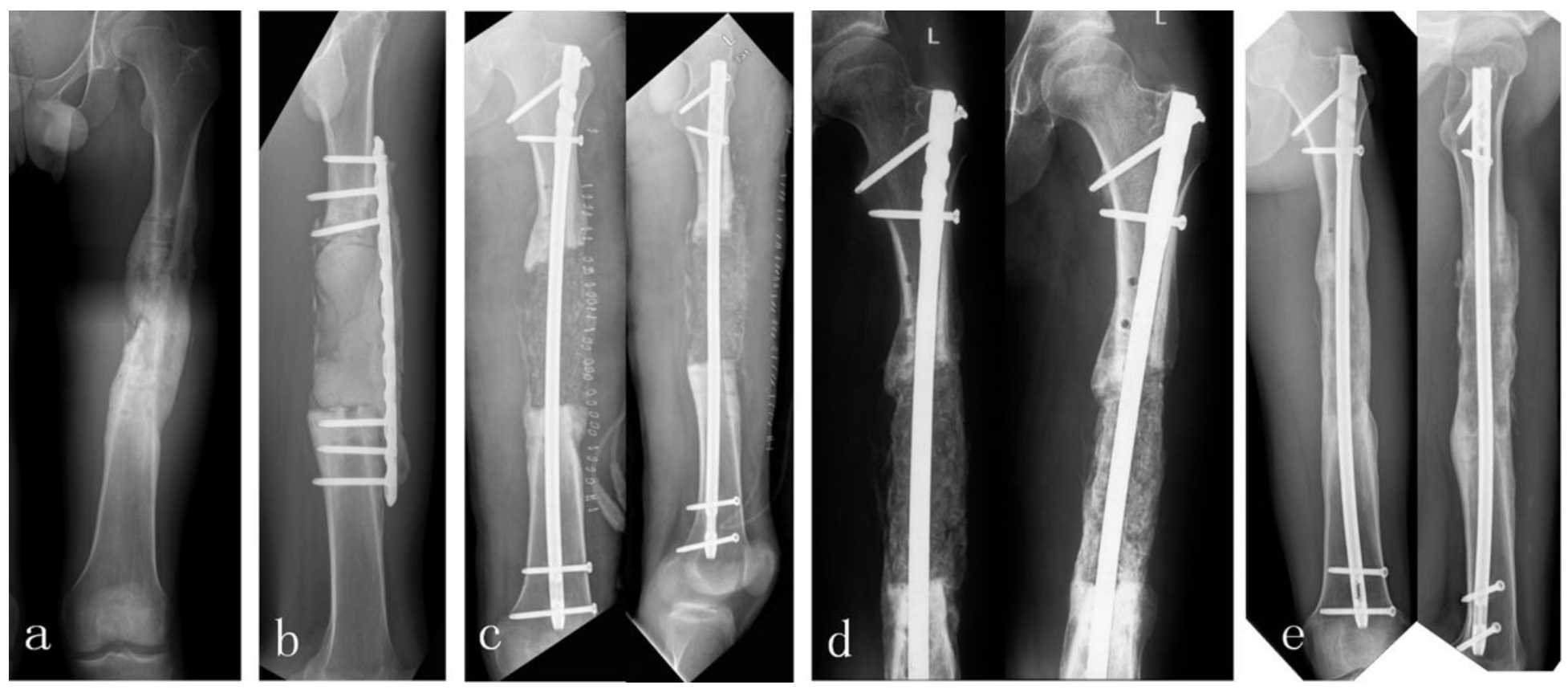
Figure 1

$21 Y$, Male, suffered from bone infection of the left femur for 2 years, the result of bacterial culture during operation was Enterobacter cloacae. a: X-Ray shows bone destruction; b: Resection of the lesion, antibiotic cement filled the defect and wrapped the plate; c: Bone graft after 8 weeks; d:Callus growth was observed after 3 months; e: Bone union after 24 months.
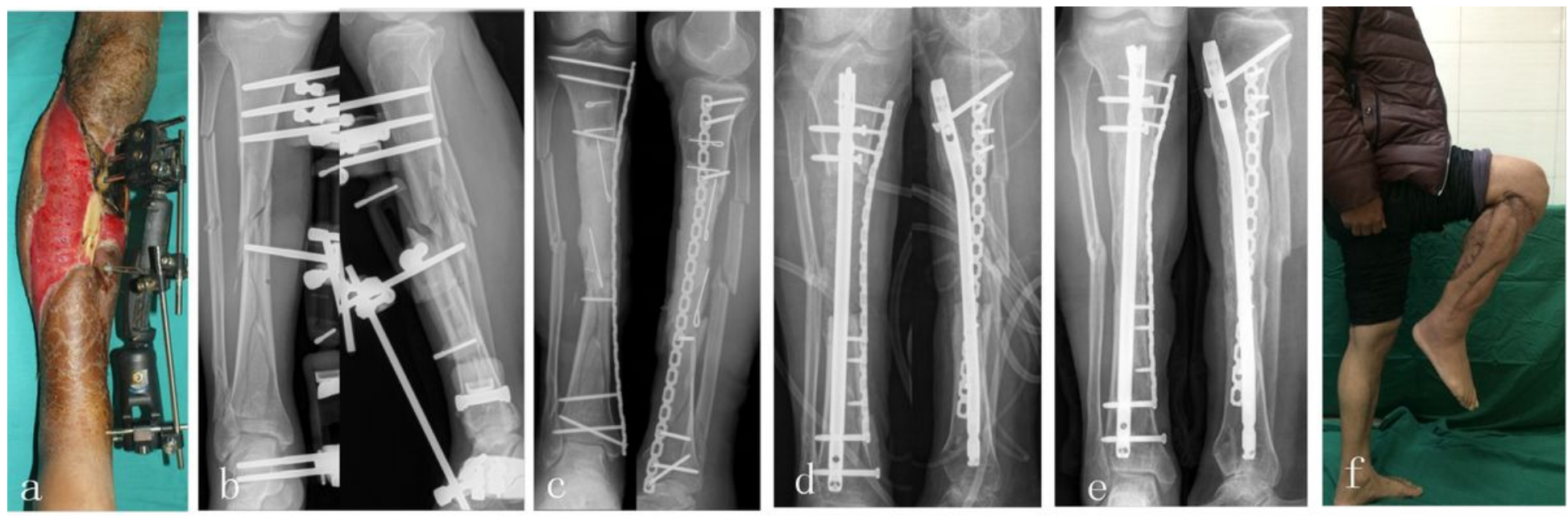

Figure 2

47Y, Male, bone infection after open injury, the culture results showed Pseudomonas aeruginosa. A:Extensive soft tissue defect and bone exposure; b:Preoperative X-Ray; $c$ : Resection of the lesion, antibiotic cement filled the defect and wrapped the plate; d: Bone graft after 4months; e: Bone union after 24 months; f: Function well.

\section{Supplementary Files}

This is a list of supplementary files associated with this preprint. Click to download.

- Table.1.doc

- Table.2.docx 